\title{
Many doctors have distorted perceptions of the value of medical tests
}

n Cite as: CMAJ 2019 February 4;191:E142-3. doi: 10.1503/cmaj.109-5695

Posted on cmajnews.com on Jan. 15, 2019.

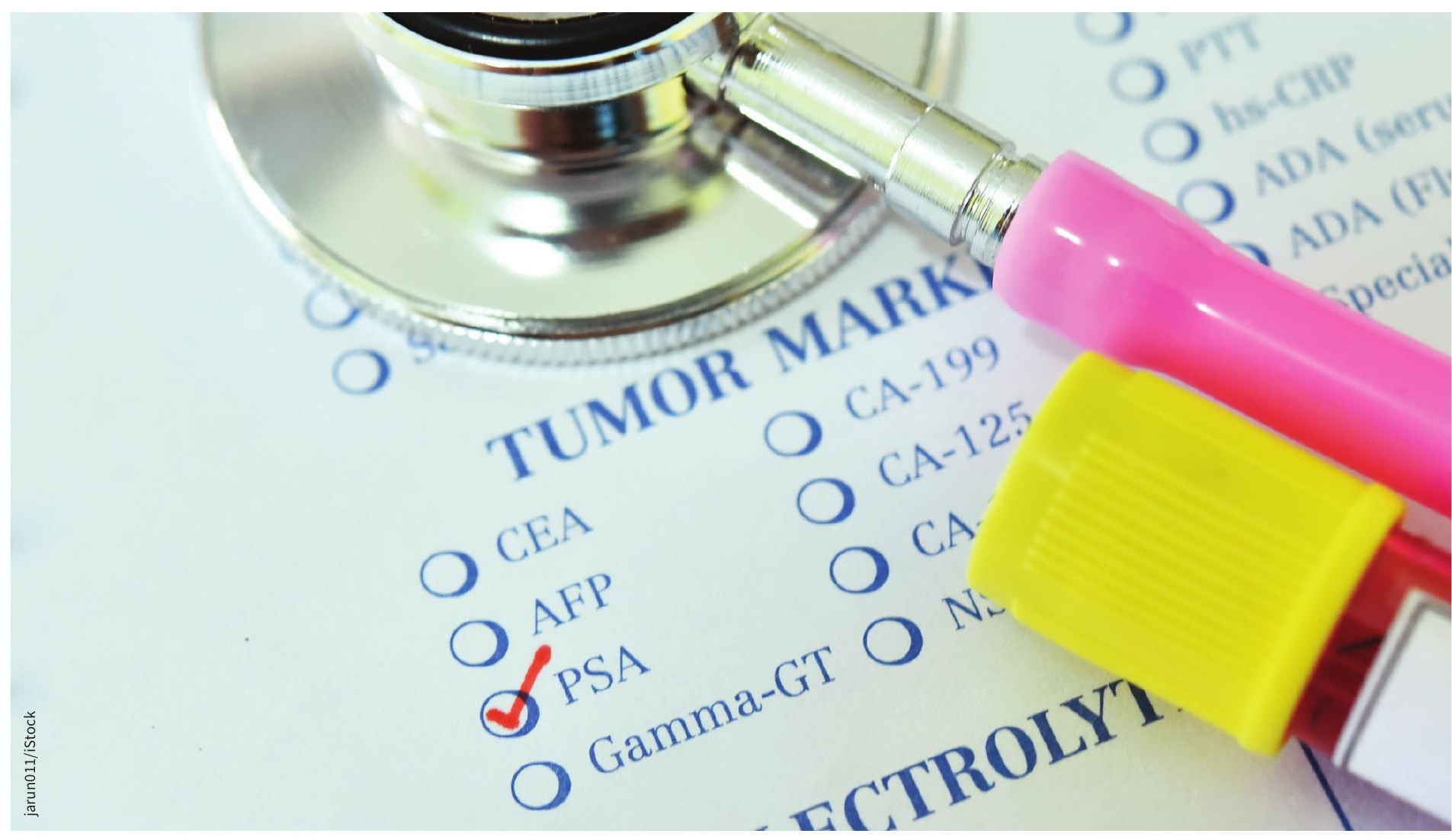

The value of the prostate-specific antigen test is a divisive topic in medicine.

n 2014, the Canadian Task Force on Preventive Health Care recommended against using the prostate-specific antigen (PSA) test to screen for prostate cancer in healthy men, concluding that it results in substantial harms via biopsies and surgeries that can lead to infections, impotence or urinary incontinence, and does not save men's lives. "Amazingly, despite thousands and thousands of patients included in randomized controlled trials, there is no mortality benefit associated with PSA testing, not a sliver," said task force member Dr. Eddy Lang, head of emergency medicine at the
University of Calgary. The task force urged doctors who offer the PSA test to discuss its "unclear benefits and substantial harms" with patients, rather than simply presenting it as a tool for cancer screening.

A few years later, a survey of family doctors in the Barrie region of Ontario discovered that the task force recommendations barely affected clinical practice. Most of the doctors responding to the survey gave the PSA test to patients and believed it was beneficial. The PSA test remains a divisive issue, and studies show many physicians' perceptions of the ben- efits and harms of any given test are distorted. They typically overestimate the chance that a patient will benefit from testing and underestimate the chance that a test will lead to harms.

"The belief in early detection is like a creed; there are people who just can't let go of that idea," said Renée Pellerin, a Canadian journalist who interviewed physicians, scientists and advocates for a 2018 book, Conspiracy of Hope, on the debates over breast cancer screening. According to Lang, many doctors have been "fooled by the false premise that 
catching cancer early makes a difference in the outcome."

The reasons doctors use harmful or lowvalue tests go beyond an attachment to cancer screening. "A test result is binary; it's positive or negative, whereas, when you're clinically assessing someone, it's not binary," said Dr. Robert Ohle, an emergency medicine physician and researcher at Health Sciences North in Sudbury, Ontario. He thinks doctors like the certainty a test appears to provide, but they often run into problems because they lack understanding of probabilities and percentages.

One example he gives is a patient arriving in an emergency room with dizziness, a common problem. To determine whether the patient is having a stroke or brain bleed, doctors often order a computed tomography (CT) scan. If the scan has no abnormal findings, they see it as changing the patient "from a low-risk to a no-risk." Yet in the case of a person presenting with dizziness, the value of a CT scan for determining whether a stroke is in progress is no better than flipping a coin. The CT scan can actually make the situation worse by falsely reassuring doctors, who may then miss a stroke.

Computed tomography also exposes patients to cancer-causing ionizing radiation, but doctors may discount that risk. Said Ohle, "It's very difficult to conceptualize what a slight increased risk of a cancer in twenty years' time means, versus the risk of missing a diagnosis today."
The problem of unnecessary CT scans is especially concerning in children's hospitals, because radiation's cancer-causing effects may be more likely in children. But doctors fear missing important injuries, so they order CT scans even when the chance of a positive finding is low.

"From a public health point of view, if we were doing millions of CT scans, there would be a potential we were creating new cancers in children," said Dr. Neil Merritt, director of pediatric trauma at the Children's Hospital of Western Ontario. Use of CT scans in children has declined over the past few years but they're still overused in the trauma setting, he said, because imaging studies without ionizing radiation, such as magnetic resonance imaging or emergency ultrasound, are harder to access.

Algorithms to assist in determining which patients actually need imaging don't always help, since physicians may not follow them, as happened with the Canadian CT Head Rule. Designed to enable emergency physicians to be more selective when referring adults with minor head injuries for $\mathrm{CT}$, it actually increased the number of scans obtained, according to a 2010 study, possibly because of emergency room crowding.

Merritt thinks the solution could be "clinical decision support" via the electronic health record. "So if you had a trauma patient roll into the bay, you'd be able to enter the information and right away determine whether or not this patient has a high risk of having some finding on their CT scan, and therefore make an informed decision." But he acknowledges that decision support at that level is in its infancy in Canada.

Another strategy is to engage and empower patients, whom doctors consider a big driver of the problem through requests for unnecessary tests and treatments. The Choosing Wisely campaign has increased doctors' awareness of the issue and is also trying to educate patients via pamphlets and signage in hospitals. Ohle thinks this could help because patients view risk differently than doctors. "Once you explain the probability of their getting harmed from the test versus the probability that they actually have the condition of interest, most patients are reasonable and they don't want to expose themselves to harm unnecessarily," he said.

Shared decision-making can be difficult to implement in a busy trauma centre, but it has a clear role in primary care. Canadian and American task forces recommend that any man considering screening for prostate cancer should have a chance to first discuss the pros and cons with a doctor. Said Lang, "If men knew what the risks were associated with [PSA] testing and how slim to nonexistent the benefits are, no man in his right mind would get tested."

Dr. Miriam Shuchman, Toronto, Ont. 